\title{
Fetal alcohol syndrome: a prospective national surveillance study
}

\author{
E J Elliott, ${ }^{1} \mathrm{~J}$ Payne, ${ }^{2}$ A Morris, ${ }^{1}$ E Haan, ${ }^{3}$ C Bower ${ }^{2}$, Paediatricians contributing to the \\ Australian Paediatric Surveillance Unit
}

\section{See Perspective, p 721}

${ }^{1}$ Discipline of Paediatrics and Child Health, University of Sydney, The Children's Hospital at Westmead and The Australian Paediatric Surveillance Unit, Westmead, NSW, Australia; ${ }^{2}$ Telethon Institute for Child Health Research, Centre for Child Health Research, The University of Western Australia, Subiaco, WA, Australia; ${ }^{3}$ Women's and Children's Hospital, Adelaide, SA, Australia

\section{Correspondence to:}

Professor Elizabeth Elliott, University of Sydney Discipline of Paediatrics and Child Health, c/o The Children's Hospital at Westmead, Locked Bag 4001, Westmead 2145, NSW, Australia; elizabe2@chw.edu.au

Accepted 8 August 2007 Published Online First 17 August 2007

\section{ABSTRACT}

Objective: To describe the epidemiology of cases of fetal alcohol syndrome (FAS) seen by Australian paediatricians. Methods: Active, national case-finding using the Australian Paediatric Surveillance Unit (APSU). Monthly reporting of incident cases aged $<15$ years by paediatricians between January 2001 and December 2004.

Results: Over 1150 paediatricians submitted reports each month to the APSU. Of 169 reported cases, 92 fulfilled the study criteria for FAS. There was a significant increase in the number of children reported each year from 2001 to 2004. Of 92 children, 53.3\% were male, $35.7 \%$ were preterm ( $<37$ weeks' gestation) and $64.6 \%$ were of low birth weight $(<2.5 \mathrm{~kg})$. Most $(94.4 \%)$ had high risk exposure to alcohol in utero and $78.3 \%$ were exposed to one or more additional drugs. The median age at diagnosis was 3.3 years (range: newborn to 11.9 years): $6.5 \%$ were diagnosed at birth and $63 \%$ by 5 years of age. Of the 92 cases, $56 \%$ had growth deficiency, $53.2 \%$ had microcephaly, $85.9 \%$ had evidence of central nervous system dysfunction, $24 \%$ had additional birth defects, $5.4 \%$ had sensorineural deafness and $4.3 \%$ had visual impairment. Of children with FAS, 65\% were Indigenous, $51 \%$ had a sibling with FAS, and only $40.2 \%$ lived with a biological parent.

Conclusion: Our data are the only prospective national data available on FAS throughout the world. These findings highlight the severity, complexity and impact of FAS, the need for effective strategies for prevention, and the necessity for education to facilitate earlier diagnosis, referral and reporting of cases.

Fetal alcohol syndrome (FAS) represents the severe end of the spectrum of structural, behavioural and neurodevelopmental abnormalities caused by exposure to alcohol in utero. A report on a series of children exposed to alcohol during pregnancy was published in $1968^{1}$ and the reproducible pattern of malformations resulting from alcohol exposure was clarified in the English medical literature in $1973 .^{2}$ Since then, a few Australian reports have been published, ${ }^{3-8}$ but none of these were national studies. Similarly, the condition is poorly described in the UK and Europe. In contrast, a wealth of literature has been published from North America, where guidelines for the diagnosis of FAS have been formulated. ${ }^{9-11}$ FAS has a huge economic and societal impact. ${ }^{12}$ By 1988 FAS was costing the US community over US\$ 4 billion per annum ${ }^{13}$ with an estimated lifetime cost per individual with FAS of US\$ 2.9 million in $2002 .^{12}$

FAS is a chronic disorder with poor prognosis. Many adolescents and adults with FAS have had disrupted education (60\%), have experienced problems with the law (60\%), drug and alcohol abuse

\section{What is already known on this topic}

- Fetal alcohol syndrome is a common cause of medical, cognitive and behavioural problems and has a huge societal and economic impact.

- Rates of alcohol use, binge drinking and drinking during pregnancy are increasing in young Australian women.

- However, no national data about fetal alcohol syndrome have been previously published in Australia.

\section{What this study adds}

- These national data on fetal alcohol syndrome (FAS) in Australia, highlight the complexity of this condition, the need for community education, prevention programs and specialised diagnostic services, and the strong over-representation of Indigenous children.

- Under-recognition of FAS is likely in Australia and may relate to paediatricians' failure to ask about alcohol use in pregnancy, lack of knowledge about the diagnosis and management of FAS, and fear of stigmatising the child and family.

(30\%) and mental health disorders (90\%), and have high rates of unemployment (90\%). ${ }^{14}$ Early diagnosis and rearing in an appropriate environment can decrease these risks fourfold. ${ }^{14}$ Australian data are required to document FAS rates, societal groups at risk, co-morbidities, and health and educational requirements. Such data are crucial to inform prevention and the development and funding of specialised diagnostic and intervention services.

We used the Australian Paediatric Surveillance Unit (APSU), ${ }^{15}$ which facilitates prospective, active, national case-finding, to ascertain newly diagnosed cases of FAS in children aged $<15$ years and to describe their epidemiology. Cases were reported each month by Australian paediatricians. A secondary objective was to educate clinicians about the clinical features of and diagnostic criteria for FAS.

\section{METHODS}

\section{Case definition}

Paediatricians were asked to report any cases of FAS diagnosed in a child aged $<15$ years between 
January 2001 and December 2004 inclusive. Before the study, paediatricians were given written information about the diagnostic criteria for FAS ${ }^{16}$ (box 1), which include exposure to alcohol in utero, characteristic craniofacial abnormalities (short palpebral fissure, long smooth philtrum, thin upper lip), prenatal and/or postnatal growth deficiency, and abnormal structure or function of the central nervous system (CNS) (including microcephaly and other structural brain abnormalities, persistent abnormal neurological signs, cognitive impairment, and speech, language, behavioural and emotional problems). The study protocol and questionnaire can be found at http://www.apsu.org.au.

Paediatricians were sent a pictorial lip-philtrum guide illustrating the facial abnormalities and describing how to measure palpebral fissure length. This guide, developed by the Washington State Fetal Alcohol Syndrome Diagnostic and Prevention Network, enables clinicians to grade the severity of facial abnormalities. ${ }^{11}$

\section{Case ascertainment and classification of cases}

Active case ascertainment facilitated by the APSU has been described. ${ }^{15}$ Practising paediatricians in Australia $(n=1154)$ receive a monthly report card (reply-paid post or email) on which they note whether or not they have diagnosed a child with FAS in the previous month. During the study period $96 \%$ ( $n=55$ 392) of all cards were returned.

Paediatricians notifying a case receive a short reply-paid questionnaire requesting partially de-identified demographic data (date of birth, age at diagnosis, sex, state/territory and postcode of residence, Indigenous status), clinical characteristics (facial features, birth defects, growth, abnormalities of the CNS, emotional, behavioural and cognitive problems), and details about management and short term outcome. Information was collected on alcohol exposure in utero, the child's mother (age, parity, use of other drugs during pregnancy, educational attainment) and family (siblings with FAS), contact with community services, use of medical and education specialists, and place of residence of the child. Paediatricians not returning monthly report cards or questionnaires were sent up to three reminders and then phoned. Using questionnaire data, we identified duplicate cases and errors (outside the age group, prevalent cases, wrong diagnosis). Confirmed cases were classified as FAS (alcohol exposure confirmed), suspected FAS (alcohol exposure not confirmed) or partial FAS (alcohol exposure confirmed), based on the Institute of Medicine criteria of $1996^{16}$ or "did not fulfil the case definition"; level of risk to the fetus was also assessed (box 1).

\section{Analysis of data}

Reported rates of FAS and birth prevalence with 95\% confidence intervals $(95 \%$ CI) were calculated using Stat-Exact. Subgroups were compared using Student t test, the Mann-Whitney U test, the $\chi^{2}$ test or Fisher's exact test. Trends were analysed using the Cochran-Armitage formula.

This study was approved by the Women's and Children's Health Service Ethics Committee, Perth, Western Australia.

\section{RESULTS}

\section{Reporting rates and case classification}

Paediatricians notified 169 FAS cases (fig 1) and returned a questionnaire providing clinical details for 159 (94\%): there were 27 reporting errors, 15 duplicate cases, 25 cases diagnosed by paediatricians as FAS but not fulfilling our case definition, and
92 classified as FAS $(n=25)$, partial FAS $(n=65)$ or suspected FAS $(n=2)$.

Of the 92 cases, 15 (16.3\%) were notified in 2001, 14 (15.2\%) in 2002, $35(38.0 \%)$ in 2003 and $28(30.4 \%)$ in 2004 ( $\chi^{2}$ trend $p=0.003)$, giving an average of 23 incident cases per year. Cases were notified from most Australian states/territories including 29 (31.5\%) from Queensland, 28 (30.4\%) from New South Wales, 12 (13\%) from Western Australia, 11 (12\%) from the Northern Territory, 6 (6.5\%) from South Australia, 5 (5.4\%) from Australian Capital Territory and 1 (1.1\%) from Victoria. Reported rates of FAS were significantly higher in Indigenous than non-Indigenous children (table 1). Birth prevalence was 0.06 per 1000 live births (95\% CI 0.04 to 0.07 ).

\section{Characteristics of children with FAS}

The median age of the children at diagnosis was 3.26 years: six were diagnosed at birth and $63 \%$ by the age of 5 years (table 1 ). One third of cases were preterm and two thirds were of low birth weight. Only $57 \%$ had all three characteristic facial features. Over half the children had growth restriction, which was evident at birth in $36 \%$.

Microcephaly in 49 (53.2\%) children was the most common structural CNS abnormality (table 1) and was noted at birth in $13(26.5 \%)$, at diagnosis in $22(44.9 \%)$ and at birth and diagnosis in $14(28.6 \%)$. We do not know the total number of children who had CNS imaging; however, five children (four with microcephaly) had one or more structural brain abnormalities.

Most children had CNS dysfunction (85.9\%). Speech and language disorders were reported in $59.8 \%$, and fine motor delay, intellectual impairment, gross motor delay and coordination problems each in about half of all children (table 1). In addition to features required for diagnosis, $24 \%$ of children had features consistent with alcohol-related birth defects. ${ }^{10}{ }^{15}$ These included musculoskeletal abnormalities (cervical rib, proximal placement of thumbs, pectus carinatum, thoracic kyphosis, talipes, spina bifida occulta, persistently patent fontanelles) in seven children, cardiovascular abnormalities (VSD, ASD, PDA, PS, PFA, vascular ring, haemangioma) in eight, renal abnormalities (horseshoe kidney, vesicoureteric reflux, renal dysplasia) in two, cleft palate in two, and microphthalmia in one.

\section{Maternal and family characteristics, fetal exposure to alcohol and other substances, and use of specialist services}

Most mothers were born in Australia: 65\% were identified as Aboriginal or Torres Strait Islander (table 2). Less than half the children with FAS lived with a biological parent. As a result, data on siblings were available for only 49 children, of whom 25 $(51 \%)$ had a sibling with FAS. Although few data were provided on maternal educational attainment, $13 \%$ of mothers were reported as having completed only primary education.

Alcohol exposure in utero was uncertain for two cases of suspected FAS: the remaining children were classified as high risk $(94.4 \%)$ or some risk $(5.4 \%)$ according to criteria in box 1. Almost $80 \%$ of children were exposed to one or more substances in addition to alcohol, commonly nicotine and marijuana (table 2). A wide range of medical, educational, psychological and community services is used by children with FAS (table 2).

\section{Behavioural and emotional problems reported in children with FAS}

One or more behavioural problems were reported in 45 (48.9\%) children and emotional problems in $26(28.3 \%)$. Table 3 lists the 
Box 1 Method used for classification of fetal alcohol

syndrome and assessment of level of risk to the fetus resulting from alcohol exposure in pregnancy ${ }^{16}$

\section{1) Classification of FAS}

Fetal alcohol syndrome - alcohol exposure confirmed

- Evidence of prenatal alcohol exposure in association with:

- all characteristic cranio-facial abnormalities,

- and prenatal and/or postnatal growth deficiency,

- and structural abnormalities or dysfunction of the central nervous system.

Suspected fetal alcohol syndrome - alcohol exposure not confirmed

- No confirmed evidence of prenatal alcohol exposure but presence of:

- all characteristic cranio-facial abnormalities,

- and prenatal and/or postnatal growth deficiency,

- and structural abnormalities or dysfunction of the central nervous system.

Partial fetal alcohol syndrome - alcohol exposure confirmed

- Evidence of prenatal alcohol exposure in association with:

- some characteristic cranio-facial abnormalities,

- and prenatal and/or postnatal growth deficiency,

- or structural abnormalities of the central nervous system,

- or dysfunction of the central nervous system.

2) Assessment of risk to the fetus

- High risk: Report from the birth mother of alcohol intake in pregnancy or directly from another individual who saw the mother drink during pregnancy. An intake of $\geqslant 4$ standard drinks in one sitting at least once weekly or binge drinking ( $>5$ standard drinks in one sitting), particularly in the first trimester of pregnancy constitutes high risk.

- Some risk: Report by the birth mother, other direct observer or reliable source of alcohol intake during pregnancy in frequencies and volumes less than the high risk category (ie, less than 4 drinks in one sitting at least once weekly but one or more drinks once per month).

- No risk: The mother reliably acknowledges no exposure to alcohol in pregnancy, or minimal exposure (ie, less than one drink once per month)

- Unknown risk: Exposure in utero is not known or information is of uncertain reliability

range of reported behavioural and emotional problems. Problems were consistent with those described in "alcoholrelated neurodevelopmental disorders"10 16 and classified according to the broad diagnostic categories in ICD-10. The most frequent externalising problems were aggression, violence, oppositional behaviour and problems with attention and hyperactivity. Internalising problems included anxiety and depression in $20.7 \%$.

\section{DISCUSSION}

This study affirms FAS as a complex, chronic disorder impacting significantly on children and families and requiring input from a range of health, community, remedial education and other services. FAS prevention must be informed by contemporary epidemiological data. Early intervention decreases long term adverse effects but requires early diagnosis. Current national data are therefore essential to inform the planning of prevention and diagnostic services. However, no such data on FAS have previously existed in Australia. Our cases were classified using

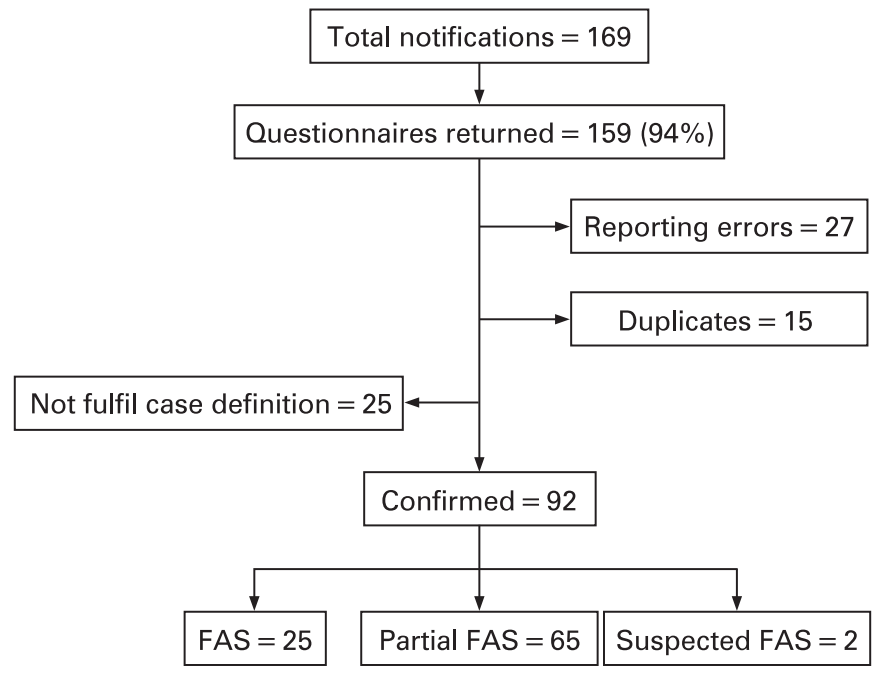

Figure 1 Notifications of fetal alcohol syndrome (FAS) to the Australian Paediatric Surveillance Unit (APSU) (2001-2004) and case classification.

strict diagnostic criteria to ensure we identified only children with FAS and not children with alcohol-related birth defects or alcohol-related neurodevelopmental disorders. Active surveillance, with reporting by the majority ( $~ 93 \%)$ of paediatricians in Australia, high monthly response rates ( $\sim 96 \%)$ to surveillance prompts, and high rates (94\%) of provision of standardised clinical data on cases ensure these data are representative of Australian paediatricians and their patients. Provision of clinical data by paediatricians actually caring for the notified child increases the likelihood of complete and accurate information.

Based on the 92 cases, the reported FAS rate per annum was 0.58 per $10^{5}$ children aged $<15$ years, or 1.14 per $10^{5}$ children aged $<5$ years. Rates were significantly higher in Indigenous children. Birth prevalence was 0.06/1000 live births. Although there are no previous national data, FAS rates have been estimated in Western Australia using two data sources (the Western Australian Birth Defects Register and the Rural Paediatric Services Database). ${ }^{6}$ The overall birth prevalence of FAS in Western Australia (0.18/1000 live births) was considered an underestimation. This consisted of $0.02 / 1000$ nonIndigenous births and 2.76/1000 Indigenous births (over 100 times the non-Indigenous rate). In a 10-year retrospective study in the Northern Territory, ${ }^{7}$ case notes were reviewed for all children with features consistent with ICD-9 and ICD-10 codes for FAS (microcephaly, fetus or newborn affected by maternal use of drugs of addiction, mental and behavioural disorders due to alcohol, or drug withdrawal syndrome in the newborn). All cases were Indigenous and the birth prevalence was 0.68 to 1.87/ 1000 live births overall and 1.7 to 4.7/1000 live Indigenous births.

Our study has limitations. One objective was to determine FAS incidence; however, adherence to strict diagnostic criteria developed at study onset necessitated exclusion of 25 children notified by paediatricians. These cases did not meet our case definition because paediatricians had insufficient information to complete questionnaires. There are several explanations for this. As many $(72 \%)$ of the children who did not fulfil the case definition did not live with their biological parents and $65 \%$ were identified as Indigenous, medical records from birth, infancy and pregnancy (including maternal alcohol consumption) were often unavailable. Nevertheless, the characteristics of excluded and included cases (growth, CNS abnormalities, exposure to other substances, and birth defects) were similar. 
Table 1 Reported rate and clinical characteristics of children with fetal alcohol syndrome

\begin{tabular}{|c|c|}
\hline & $\begin{array}{l}\text { Reported rate per } 10^{5} \\
\text { per annum }(95 \% \mathrm{Cl}) \dagger\end{array}$ \\
\hline Children aged $<15$ years at diagnosis (total population) & 0.58 (0.46 to 0.71$)$ \\
\hline Non-Indigenous population & $0.18(0.12$ to 0.27$)$ \\
\hline Indigenous population $(p<0.001)$ & $8.11(6.19$ to 10.44$)$ \\
\hline Children aged $<5$ years at diagnosis (total population) & $1.14(0.87$ to 1.47$)$ \\
\hline Non-Indigenous population & $0.37(0.22$ to 0.59$)$ \\
\hline \multirow[t]{2}{*}{ Indigenous population $(p<0.001)$} & 14.60 (10.22 to 20.22$)$ \\
\hline & $\begin{array}{l}\text { Clinical } \\
\text { characteristics } \\
(\mathrm{n}=92)^{*}, \mathrm{n}(\%)\end{array}$ \\
\hline Sex (male) & $49(53.3)$ \\
\hline Gestation $<37$ weeks $(n=84)$ & $30(35.7)$ \\
\hline Mean (SD) gestation (weeks) & $37.4(2.68)$ \\
\hline Birth weight $<2.5 \mathrm{~kg}(\mathrm{n}=82)$ & $53(64.6)$ \\
\hline Mean (SD) birth weight (kg) & $2.36(0.53)$ \\
\hline Median age at FAS diagnosis in years (range) & 3.26 (newborn to 11.9) \\
\hline Diagnosis at birth & $6(6.5)$ \\
\hline Diagnosis at age $<1$ year & $22(23.9)$ \\
\hline Diagnosis at age $<2$ years & $36(39.1)$ \\
\hline Diagnosis at age $<5$ years & $58(63)$ \\
\hline \multicolumn{2}{|l|}{ Diagnostic features } \\
\hline Facial features (any) & $92(100)$ \\
\hline Smooth philtrum & 89 (96.7) \\
\hline Thin upper lip & $87(94.6)$ \\
\hline Short palpebral fissure & $56(60.9)$ \\
\hline All of the above features & $53(57)$ \\
\hline Growth restriction $(\mathrm{n}=91)$ & $51(56.0)$ \\
\hline Normal & $40(44.0)$ \\
\hline Prenatal only & $22(24.2)$ \\
\hline Postnatal only & $18(19.8)$ \\
\hline Both prenatal and postnatal & $11(12.1)$ \\
\hline CNS structural abnormality (any) & $50(54.3)$ \\
\hline Microcephaly ( $\mathrm{HC}<3$ rd percentile) alone & $45(48.9)$ \\
\hline Microcephaly plus other structural defect $\$$ & $4(4.3)$ \\
\hline Structural defect alone (agenesis corpus callosum) & $1(1.1)$ \\
\hline CNS dysfunction & $79(85.9)$ \\
\hline Speech/language disorder & $55(59.8)$ \\
\hline Fine motor delay & $46(50.0)$ \\
\hline Intellectual impairment & 45 (48.9) \\
\hline Behavioural problems & $45(48.9)$ \\
\hline Gross motor delay & $33(35.9)$ \\
\hline Poor coordination & $36(39.1)$ \\
\hline Emotional problems & $26(28.3)$ \\
\hline Abnormal muscle tone & $20(21.7)$ \\
\hline Abnormal deep tendon reflexes & $12(13)$ \\
\hline Refractory error & $7(7.6)$ \\
\hline Seizure disorder & $6(6.5)$ \\
\hline Tremor & $6(6.5)$ \\
\hline Sensorineural hearing loss & $5(5.4)$ \\
\hline Visual impairment & $4(4.3)$ \\
\hline Nystagmus & $2(2.2)$ \\
\hline
\end{tabular}

${ }^{*} \mathrm{n}=92$ unless otherwise stated.

$\uparrow$ Rates calculated using population estimates for Aboriginal and Torres Strait Islander and non-Indigenous populations from the Australian Bureau of Statistics for the years of the study. $p<0.001$ indicates the difference in reported rates between Indigenous and non-Indigenous children in each age group.

Structural defects of the central nervous system (CNS) included agenesis of the corpus callosum, absent pituitary stalk, thalamic cyst, cerebral atrophy, subcortical gliosis, delayed white matter myelination and abnormal cerebellum.

$\mathrm{HC}$, head circumference.

Furthermore, $52 \%$ of excluded cases had a sibling with FAS, suggesting that these were likely true cases and that we have underestimated incidence.
Table 2 Maternal and family characteristics, exposure to alcohol and other substances in utero' and use of specialist medical and other services $^{2}$ in 92 children with fetal alcohol syndrome (FAS)

\begin{tabular}{ll}
\hline & Cases of FAS, \\
$\mathbf{n}(\%)$
\end{tabular}

*Includes benzodiazepines, naltrexone, epilim, morphine, pethidine, labetolol, nortriptyline, largactil, solvents and methadone.

†Early intervention, speech therapy, occupational therapy, lactation consultant, ENT surgeon and Aboriginal Medical Service.

Another limitation of the study was that the diagnostic criteria for partial FAS given to paediatricians stated "... and structural abnormalities or dysfunction of the central nervous system" instead of "... or structural abnormalities or dysfunction of the central nervous system". Thus it is possible that some paediatricians may have under-reported cases of partial FAS, although we believe this was unlikely. The data for partial FAS were subsequently coded in accordance with the classification of FAS (box 1).

A further difficulty in accurately estimating FAS rates is that clinicians often find it difficult to make a clinical diagnosis of $\mathrm{FAS}^{17}{ }^{18}$ and about $50 \%$ of paediatricians in Western Australia agree that FAS is more difficult to diagnose in Indigenous children. ${ }^{18}$ FAS facial features become less obvious with age and although the median age at reporting to APSU in patients who did not fulfil the case definition was 2.8 years, the upper limit was 12.6 years. Since our study commenced, the Institute of Medicine criteria have been revised" to make them "more specific and clinically applicable in general paediatric practice". 
Table 3 Behavioural and emotional problems reported in children with fetal alcohol syndrome (FAS)*

\begin{tabular}{lc}
\hline & $\begin{array}{l}\text { Cases of FAS } \\
\text { (n = 92), } \mathbf{n}(\%)\end{array}$ \\
\hline Externalising problems & \\
Oppositional defiant disorder & \\
Aggression/violence & $14(15.2)$ \\
Oppositional behaviour & $7(7.6)$ \\
Disruptive behaviour & $1(1.1)$ \\
Disobedient/uncooperative & $1(1.1)$ \\
Acting out & $1(1.1)$ \\
Attention/hyperactivity disorders & \\
Attention deficit hyperactivity disorder & $11(12)$ \\
Hyperactivity & $11(12)$ \\
Attention/concentration problems & $7(7.6)$ \\
Neonatal irritability (? alcohol withdrawal) & $2(2.2)$ \\
Impulsivity & $2(2.2)$ \\
Irritability & $1(1.1)$ \\
Internalising problems & \\
Anxiety & $2(2.2)$ \\
Withdrawn/socially isolated & $5(5.4)$ \\
Easily distressed, emotional fragility, emotional lability & $4(4.4)$ \\
Problems adjusting to new carers & $3(3.3)$ \\
Panic attacks & $2(2.2)$ \\
Solitary repetitive behaviour & $1(1.1)$ \\
Post-traumatic stress disorder & $1(1.1)$ \\
Depression & $1(1.1)$ \\
Abnormal eating behaviours & $1(1.1)$ \\
School problems & \\
Bullied/teased, school absenteeism & $4(4.4)$ \\
Other & \\
Autistic spectrum disorders, self injury, inappropriate & $5(5.5)$ \\
sexual behaviours & \\
\hline Behalo & \\
\hline
\end{tabular}

*Behavioural and emotional symptoms and conditions have been classified according to the broad diagnostic categories used in ICD-10. Some children had more that one problem reported.

Another difficulty in estimating rates relates to lack of access to paediatricians, especially by Indigenous people in remote, rural areas. Despite this potential limitation, Indigenous children were highly over-represented, constituting $65.2 \%$ of all cases reported (the proportion of Indigenous children in Australia in 2004 was <5\%). Also, the Northern Territory, with $1 \%$ of the Australian population but a relatively higher proportion of Indigenous people, was over-represented in our study with $12 \%$ of reported FAS cases.

Under-reporting may also be a consideration: the proportion of cases (1\%) reported from Victoria (24\% of Australia's population) was lower than expected. Personal communication with specialist paediatric services confirms that few cases were seen, suggesting the problem may be lack of recognition and referral by non-specialists. This is supported by our survey of health professionals (Aboriginal health workers, allied health professionals, community nurses, general practitioners and obstetricians) in Western Australia. ${ }^{17}$ Only $12 \%$ identified the diagnostic features, most had never made the diagnosis, and only $2 \%$ felt very prepared to deal with FAS.

Under-diagnosis by paediatricians might also be relevant. In our survey of Western Australian paediatricians, ${ }^{18}$ only $23 \%$ routinely asked about alcohol use in a pregnancy history and only $19 \%$ correctly identified the diagnostic features of FAS. In that survey, ${ }^{18} 76.5 \%$ of paediatricians had suspected but not diagnosed FAS and $12.1 \%$ had on occasion been convinced of but not recorded the diagnosis. A secondary objective of the APSU study was to educate paediatricians about FAS and, in the
Western Australia survey, ${ }^{18} 32 \%$ of paediatricians said APSU information had increased their awareness of FAS. The significant increase in cases reported over the APSU study period suggests that recognition improved. However, 9\% of Western Australia paediatricians said they had diagnosed a child with FAS during 2001-2004 but not notified the APSU. Failure to diagnose and report may be due to fear of stigmatisation of the child or family (expressed by $69.6 \%$ of paediatricians ${ }^{18}$ and $53 \%$ of health professionals ${ }^{17}$ ) and lack of knowledge about how to refer and manage children. Most health professionals surveyed requested educational materials for themselves and their patients. ${ }^{17} 18$

Early diagnosis decreases problems in adults with FAS, ${ }^{14}$ but in our study diagnosis in some children was as late as 12 years and $37 \%$ remained undiagnosed at 5 years. Children with FAS demonstrated the full spectrum of abnormalities previously reported. ${ }^{10}$ Although many were still very young $23.9 \%$ were less than 1 year old), 48.9\% had intellectual impairment. Language and speech problems (59.8\%), and sensorineural hearing problems (5.4\%) influence educational attainment. Problems with executive function are important in $\mathrm{FAS}^{10}$; however, these are less obvious in children, may not be evident unless sought with special testing, and were not specifically reported.

FAS is a diagnosis of exclusion and a careful family history and exclusion of genetic conditions is important. ${ }^{10}{ }^{11}$ Ideally, children should be diagnosed in multi-disciplinary clinics which include developmental paediatricians, psychologists and clinical geneticists. ${ }^{9}$ However, no such services exist in Australia. In addition, early diagnosis allows for prevention within families. A salutary finding in our study was that where information was available, $51 \%$ of cases were reported to have a sibling with FAS, indicating missed opportunities for prevention. This proportion may be high because of the increased probability of diagnosing a condition if other family members are affected. It is of additional concern that nearly $80 \%$ of children with FAS were exposed in utero to at least one drug in addition to alcohol. Opportunities for maternal education about the effects of substance use in pregnancy may be limited because only $40 \%$ of children with FAS lived with a biological parent.

We have proposed a causal pathway for $\mathrm{FAS}^{8}$ and identified some of the societal and individual factors that contribute to FAS and opportunities for prevention. Prevention will require clear government policy regarding alcohol use in pregnancy and co-operation across government departments including health, education, housing, legal and community services. In Australia, alcohol policy varies between states and territories ${ }^{19}$ and National Health and Medical Research Alcohol guidelines ${ }^{20}$ are currently under review. Prevention in high risk, including Indigenous, populations will require the development and evaluation of culturally appropriate and language specific tools.

For the reasons outlined above, we believe FAS incidence is under-estimated in Australia. Nevertheless, rates are likely to be maintained considering that rates of drinking, particularly binge drinking, are on the increase in young women, rates of drinking in pregnancy are high, and half of pregnancies are unplanned. Apart from the financial costs, the societal impact of FAS is considerable. Our data will focus attention on this condition and inform resource allocation, prevention and future research.

Acknowledgements: We thank all child health specialists who participate in APSU surveillance and particularly those who reported cases to this study. We thank the Australian Paediatric Surveillance Unit (APSU) staff and Scientific Review Panel, and Dr Alison Laversha and Dr Ingrid Bucens for their help in developing the study proposal.

Funding: This study was funded the Health Promotion Foundation of Western Australia (Healthway Project Grant 10563) and the National Health and Medical Research Council (NH\&MRC) Program Grants 003209 and 353514, and NH\&MRC 
Fellowships (CB: 172303 and 353625 and EE: 457084). The APSU is a Unit of the Division of Paediatrics and Child Health, Royal Australasian College of Physicians and is funded by the Department of Health and Ageing, Faculty of Medicine at the University of Sydney, and an NHMRC Enabling Grant (402784).

Competing interests: Supporting sources had no role in the study design, data collection, analysis and interpretation or writing of the article. The supporting sources did not control or influence the decision to submit the final manuscript for publication.

\section{REFERENCES}

1. Lemoine $\mathbf{P}$, Harousseau $\mathrm{H}$, Borteyru JP, et al. Les enfants des parents alcoholiques: anomalies observees a propos de 127 cases. Ouest Med 1968;8:476-82.

2. Jones KL, Smith DW, Ulleland CN, et al. Pattern of malformation in offspring of chronic alcoholic mothers. Lancet 1973;1:1267-71.

3. Collins E, Turner G. Six children affected by maternal alcoholism. Med J Aust 1978;30:606-8.

4. Walpole JR, Hockey A. Fetal alcohol syndrome: implications to family and society in Australia. Aust Paediatr J 1980;16:101-5.

5. Lipson AH, Walsh DA, Webster WS. Fetal alcohol syndrome: a great imitator. Med J Aust 1984;1:266-9.

6. Bower C, Silva D, Henderson TR, et al. Ascertainment of birth defects: the effect on completeness of adding a new source of data. J Paediatr Child Health 2000;36:574-6.

7. Harris $\mathbf{K}$, Bucens I. Prevalence of fetal alcohol syndrome in the Top End of the Northern Territory. J Paediatr Child Health 2003;39:528-33.

8. Elliott EJ, Bower C. FAS in Australia: fact of fiction? J Paediatr Child Health 2004:40:8-10

9. Chudley AE, Conry J, Cook JL, et al. Fetal alcohol spectrum disorder: Canadian guidelines for diagnosis. CMAJ 2005;172(5 Suppl):S1-S21.
10. Hoyme $\mathbf{H E}$, May PA, Kalberg WO, et al. A practical approach to diagnosis of fetal alcohol spectrum disorders: clarification of the 1996 Institute of Medicine criteria. Pediatrics 2005;115:39-47

11. Astley SJ, Clare SK. Diagnosing the full spectrum of fetal alcohol-exposed individuals: introducing the 4-digit diagnostic code. Alcohol Alcohol 2000;35:400-10.

12. Lupton C, Burd L, Harwood R. Cost of fetal alcohol spectrum disorders. Am J Med Genet 2004:127C:42-50.

13. Harwood H. Updating estimates of the economic costs of alcohol abuse in the United States: estimates, updated methods, and data: report prepared by the Lewin group Bethesda, MD: National Institute on Alcohol Abuse and Alcoholism, 2000.

14. Streissguth AP, Bookstein FL, Barr HM, et al. Risk factors for adverse life outcomes in fetal alcohol syndrome and fetal alcohol effects. J Dev Behav Pediatr 2004;25(4):228-38.

15. Morris A, Ridley G, Elliott E, on behalf of the APSU staff, investigators and all contributing paediatricians. The Australian Paediatric Surveillance Unit: Progress Report. J Paediatr Child Health 2002;38:8-15.

16. Stratton KR, Howe CJ, Battaglia FC, eds. Fetal alcohol syndrome: diagnosis, epidemiology, prevention and treatment. Washington, DC: National Academy Press, 1996.

17. Payne J, Elliott EJ, D’Antoine $\mathrm{H}$, et al. Health professionals' knowledge, practice and opinions about fetal alcohol syndrome and alcohol consumption in pregnancy. Aust N Z J Public Health 2005;29(6):559-64.

18. Elliott EJ, Payne J, Haan E, et al. Diagnosis of fetal alcohol syndrome and alcohol use in pregnancy: a survey of paediatricians' knowledge, attitudes and practice. J Paediatr Child Health 2006;42:698-703.

19. O'Leary CM, Elliott EJ, Heuzenroeder L, et al. A review of policies on alcohol use during pregnancy in Australia and other English-speaking countries, 2006. Med J Aust 2007; 186(9):466-71.

20. National Health and Medical Research Council. Australian alcohol guidelines: health risks and benefits. National Health and Medical Research Council, 2001. Available at http://www.nhmrc.gov.au/publications/synopses/ds9syn.htm (accessed 26 April 2008)

BNF for Children 2007-Easing shared care prescribing

Facilitating seamless care of children between hospital and the community Independent research shows that BNFC is improving the quality of prescribing for children under shared care arrangements between primary and secondary care.

For more information visit bnfc.org.

To order go to www.pharmpress.com. 